\title{
A UNIFIED THEORY FOR REAL VS. COMPLEX RATIONAL CHEBYSHEV APPROXIMATION ON AN INTERVAL
}

\author{
ARDEN RUTTAN AND RICHARD S. VARGA
}

\begin{abstract}
A unified approach is presented for determining all the constants $\gamma_{m, n}(m \geq 0, n \geq 0)$ which occur in the study of real vs. complex rational Chebyshev approximation on an interval. In particular, it is shown that $\gamma_{m, m+2}=1 / 3(m \geq 0)$, a problem which had remained open.
\end{abstract}

\section{INTRODUCTION}

Let $\pi_{m}^{r}$ and $\pi_{m}^{c}$ denote, respectively, the sets of polynomials of degree at most $m$, with real and complex coefficients. For any pair $(m, n)$ of nonnegative integers, $\pi_{m, n}^{r}$ denotes the set of rational functions of the form $p(x) / q(x)$, where $p \in \pi_{m}^{r}$ and $q \in \pi_{n}^{r}$, and we define $\pi_{m, n}^{c}$ analogously as the set of rational functions of the form $p(x) / q(x)$ where $p \in \pi_{m}^{c}$ and $q \in \pi_{n}^{c}$. Let $\|\cdot\|_{I}$ denote the supremum norm on $[-1,1]$, i.e., $\|f\|_{I}:=\sup _{x \in[-1,1]}|f(x)|$. If $C^{r}[-1,1]$ denotes the set of all continuous real-valued functions on $[-1,1]$, then, for $f$ in $C^{r}[-1,1]$, we set

$$
\begin{aligned}
& E_{m, n}^{r}(f):=\inf \left\{\|f-g\|: g \in \pi_{m, n}^{r}\right\}, \\
& E_{m, n}^{c}(f):=\inf \left\{\|f-g\|: g \in \pi_{m, n}^{c}\right\} .
\end{aligned}
$$

For $f \in C^{r}[-1,1]$, it is well known that there exist functions $h \in \pi_{m, n}^{r}$ and $g \in \pi_{m, n}^{c}$ satisfying $E_{m, n}^{r}(f)=\|f-h\|_{I}$ and $E_{m, n}^{c}(f)=\|f-g\|_{I}$. In fact, $h$ can be characterized by the length of the alternation set of $f-h$ (cf. Meinardus [2, p. 162]). Less is known about the $g$ for which $E_{m, n}^{c}(f)=\|f-g\|_{I}$. Since $\pi_{m, n}^{r} \subseteq \pi_{m, n}^{c}$, then evidently $E_{m, n}^{c}(f) \leq E_{m, n}^{r}(f)$, but it is not obvious that strict inequality can hold. What is surprizing here is that, for each $m \geq 0$ and $n \geq 1$, there is a real continuous function $f$ on the real interval $[-1,+1]$ for which

$$
E_{m, n}^{c}(f) / E_{m, n}^{r}(f)<1 .
$$

Received by the editors October 1, 1987.

1980 Mathematics Subject Classification (1985 Revision). Primary 41A20; Secondary 41A50.

Key words and phrases. Rational functions, best uniform approximation, real vs. complex approximation of real functions, alternation sets.

Research supported by the Air Force Office of Scientific Research. 
(For a recent treatment of this, which covers the early contributions of A. A. Gončar, K. N. Lungu, and Saff and Varga, see [6, Chapter 5].)

Saff and Varga [4] raised the question as to how small the ratio $E_{m, n}^{c}(f) / E_{m, n}^{r}(f)$ can be for a fixed integer pair $(m, n)$. More precisely, they asked which values the numbers $\gamma_{m, n}$ take on, where

$$
\gamma_{m, n}:=\inf \left\{E_{m, n}^{c}(f) / E_{m, n}^{r}(f): f \in C^{r}[-1,1] \backslash \pi_{m, n}^{r}\right\} .
$$

Three recent papers have described the behavior of $\gamma_{m, n}$ in terms of $(m, n)$. First, Trefethen and Gutknecht [5] established, by means of a direct construction, the surprising result that

$$
\gamma_{m, n}=0, \text { for each pair }(m, n) \text { of nonnegative integers with }
$$

$$
n \geq m+3
$$

Next, Levin [1] established the complementary result that

(1.5) $\gamma_{m, n}=1 / 2$, for each pair $(m, n)$ of nonnegative integers with

$$
m+1 \geq n \geq 1 \text {. }
$$

Levin's proof of (1.5) consisted of a direct construction to show that $\gamma_{m, n} \leq \frac{1}{2}$, and an algebraic method to show that $\gamma_{m, n}<\frac{1}{2}$ was impossible for $m+1 \geq$ $n \geq 1$. The results of (1.4) and (1.5) leave open only the case $\gamma_{m, m+2}(m \geq 0)$. For this case, Ruttan and Varga [3], also by means of a direct construction, have more recently shown that

$$
\gamma_{m, m+2} \leq 1 / 3 \quad(m \geq 0) .
$$

This result, however, leaves open the question of the actual values of $\gamma_{m, m+2}, m \geq$ 0 , allowing speculation that perhaps $\gamma_{m, m+2}$ might be zero or even that $\gamma_{m, m+2}$ might take on different values as $m$ varies.

Our object here is to complete this topic by showing that

$$
\gamma_{m, m+2}=1 / 3 \quad(m \geq 0) \text {. }
$$

In the process of establishing (1.7), we develop two results for general complex rational functions which provide a unified approach to the problem of determining the values of $\gamma_{m, n}$.

\section{UPPER BOUNDS FOR $\gamma_{m, n}$}

Table 1 lists the values of $\gamma_{m, n}$ established in [5] $(n \geq m+3)$ and in [1] $(1 \leq n \leq m+1)$, together with the values of $\gamma_{m, n}(n=m+2)$ which follow from [3] and the results to be developed below. Evidently, $\gamma_{m, n}$ takes on only four distinct values: $0,1 / 3,1 / 2$, and 1 . The value 1 occurs only when $n=0$ and is a consequence of the well known fact that the best uniform approximant, from $\pi_{m, 0}^{c}$, of any real-valued continuous function on $[-1,1]$ is a real polynomial, whence $E_{m, n}^{r}(f)=E_{m, n}^{r}(f)$. The remaining values $0,1 / 3$, and $1 / 2$ occur in 


\begin{tabular}{l|c|c|c|c|c|c|} 
& 0 & 1 & 2 & 3 & 4 & 5 \\
\cline { 2 - 7 } 0 & 1 & 1 & 1 & 1 & $\ldots$ & $\ldots$ \\
\cline { 2 - 7 } 1 & $1 / 2$ & $1 / 2$ & $1 / 2$ & $1 / 2$ & $\ldots$ & $\ldots$ \\
\cline { 2 - 7 } 2 & $1 / 3$ & $1 / 2$ & $1 / 2$ & $1 / 2$ & $\ddots$ & $\ddots$ \\
\cline { 2 - 7 } 3 & 0 & $1 / 3$ & $1 / 2$ & $1 / 2$ & $\ddots$ & $\ddots$ \\
\hline 4 & 0 & 0 & $1 / 3$ & $1 / 2$ & $\ddots$ & $\ddots$ \\
\hline & 0 & 0 & 0 & $1 / 3$ & $\ddots$ & $\ddots$ \\
\hline & 0 & 0 & 0 & 0 & $\ddots$ & $\ddots$ \\
\hline & $\vdots$ & $\vdots$ & $\vdots$ & $\vdots$ & $\ddots$ & $\ddots$ \\
\cline { 2 - 7 } & & & & &
\end{tabular}

TABLE 1. Values of $\gamma_{m, n}(m \geq 0 ; n \geq 0)$

the regions $R_{1}:=\{(m, n): n \geq m+3\}, R_{2}:=\{(m, n): n=m+2\}$, and $R_{3}:=\{(m, n): 1 \leq n \leq m+1\}$, respectively, of Table 1 .

In establishing the sharp upper bounds for $\gamma_{m, n}$ for a given region $R_{i}$, $i=1,2$ or 3 , the aforementioned authors constructed families of functions $\mathscr{F}(m, n, \varepsilon) \subseteq C^{r}[-1,1] \backslash \pi_{m, n}^{r}$, where $(m, n) \in R_{i}$ and where $\varepsilon>0$, with the property that

$$
\gamma_{m, n}=\inf \left\{E_{m, n}^{c}(f) / E_{m, n}^{r}(f): f \in \mathscr{F}(m, n, \varepsilon) \text { and } \varepsilon>0\right\} .
$$

In this section, we give a unified approach to calculating a sharp upper bound for $\gamma_{m, n}$ in each of the regions $R_{1}, R_{2}$, and $R_{3}$ of Table 1 . In addition to providing a consistent framework for calculating upper bounds of $\gamma_{m, n}$, the details presented below also provide the foundation required for the sharpness results given in Theorem 4 .

Our first result provides a new tool for obtaining upper bounds for $\gamma_{m, n}$.

Proposition 1. For a fixed pair $(m, n)$ of nonnegative integers, let

$$
\phi \in\left(\pi_{m, n}^{c} \backslash \pi_{m, n}^{r}\right) \cap C^{r}[-1,1],
$$

and let $S$ be a continuous real-valued function on $[-1,1]$ for which there are $L \geq m+2$ distinct points $\left\{x_{j}\right\}_{j=1}^{L}$, with $-1 \leq x_{1}<x_{2}<\cdots<x_{L} \leq 1$, such that

$$
(-1)^{j} \delta\left(S\left(x_{j}\right)+\operatorname{Re} \phi\left(x_{j}\right)\right)>0 \quad(j=1,2, \ldots, L),
$$

where $\delta$ is a constant which is either +1 or -1 . Then,

$$
\gamma_{m, n} \leq\|S-i \operatorname{Im} \phi\|_{I} / M,
$$


where

$$
M:=\min _{1 \leq j \leq L}\left|S\left(x_{j}\right)+\operatorname{Re} \phi\left(x_{j}\right)\right| .
$$

Proof. Set $f(x):=S(x)+\operatorname{Re} \phi(x)$. Then, as condition (2.1) states that the error function for the zero approximation to $f$ oscillates in $L \geq m+2$ points, the de la Vallée Poussin Theorem [2, p. 83] gives (cf. (2.3)) that $E_{m, n}^{r}(f) \geq M$. But as $E_{m, n}^{c}(f) \leq\|f-\phi\|_{I}=\|S-i \operatorname{Im} \phi\|_{I}$, we must have from (1.3) that $\gamma_{m, n} \leq\|S-i \operatorname{Im} \phi\|_{I} / M$.

Given a pair of nonnegative integers $(m, n)$ with $n \geq 1$, Proposition 1 suggests a procedure for finding a sequence of functions $\left\{f_{\varepsilon}\right\} \subseteq C^{r}[-1,1] \backslash \pi_{m, n}^{r}$ for which $E_{m, n}^{c}\left(f_{\varepsilon}\right) / E_{m, n}^{r}\left(f_{\varepsilon}\right)$ is minimized. One first chooses a continuous rational function $\phi_{\varepsilon} \in \pi_{m, n}^{c} \backslash \pi_{m, n}^{r}$ on $[-1,1]$ with the property that $\operatorname{Re} \phi_{\varepsilon}(x)$ has at least $m+1$ sign changes in $[-1,1]$ and for which $\left\|\operatorname{Im} \phi_{\varepsilon}\right\|_{I}$ is small. Such a function may be obtained (see Theorems 2, 3, and 4 below) by placing, in an astute manner, the zeros and poles of $\phi_{\varepsilon}$ near the interval $[-1,1]$. Suppose $(-1)^{j} \operatorname{Re} \phi_{\varepsilon}\left(x_{j}\right)>0(j=1,2, \ldots, m+2)$, where $-1 \leq x_{1}<\cdots<x_{m+2} \leq 1$. The function $S_{\varepsilon}$ is then chosen so that

$$
\begin{gathered}
\operatorname{sgn} S_{\varepsilon}\left(x_{j}\right)=\operatorname{sgn} \operatorname{Re} \phi_{\varepsilon}\left(x_{j}\right) \quad(j=1,2, \ldots, m+2), \\
\left|S_{\varepsilon}\left(x_{j}\right)-i \operatorname{Im} \phi_{\varepsilon}\left(x_{j}\right)\right| \approx\left\|\operatorname{Im} \phi_{\varepsilon}\right\|_{I} \quad(j=1,2, \cdots, m+2),
\end{gathered}
$$

and

$$
S_{\varepsilon}(x)=0, \text { for } x \notin \bigcup_{j=1}^{m+2}\left(x_{j}-\varepsilon, x_{j}+\varepsilon\right) \text {, for some sufficiently small } \varepsilon>0
$$

The condition of (2.4) is used to make

$$
M:=\min _{1 \leq j \leq m+2}\left\{\left|S_{\varepsilon}\left(x_{j}\right)+\operatorname{Re} \phi\left(x_{j}\right)\right|\right\}
$$

as large as possible, while conditions (2.5) and (2.6) are used to guarantee that $\left\|S_{\varepsilon}-i \operatorname{Im} \phi_{\varepsilon}\right\|_{I} \approx\left\|\operatorname{Im} \phi_{\varepsilon}\right\|_{I}$. These choices make the ratio $\left\|S_{\varepsilon}-i \operatorname{Im} \phi_{\varepsilon}\right\|_{I} / M$ in (2.2) nearly as small as possible.

As a concrete example of the above procedure, consider the integer pair $(0,2)$ and, for any $\varepsilon>0$ sufficiently small, set

$$
\begin{aligned}
& \phi_{\varepsilon}(x):=\frac{2 \varepsilon i}{3}\left[\frac{1}{x+1-i \varepsilon}-\frac{1}{x-1-i \varepsilon}\right], \\
& h(x):= \begin{cases}\frac{1-x^{2}}{1+x^{2}}, & x \in[-1,1] \\
0 & \text { otherwise }\end{cases}
\end{aligned}
$$

and

$$
S_{\varepsilon}(x):=\frac{1}{3}\left[h\left(\frac{x-1}{\varepsilon}\right)-h\left(\frac{x+1}{\varepsilon}\right)\right]
$$


The function $\phi_{\varepsilon}(x)$, an element of $\pi_{0,2}^{c} \backslash \pi_{0,2}^{r}$, can be verified to satisfy

$$
\operatorname{Re} \phi_{\varepsilon}(-1)=-\frac{2}{3}+O\left(\varepsilon^{2}\right)<0, \quad \text { and } \quad \operatorname{Re} \phi_{\varepsilon}(1)=\frac{2}{3}+O\left(\varepsilon^{2}\right)>0,
$$

for all $\varepsilon>0$ sufficiently small. Next, on setting $x_{1}:=-1$ and $x_{2}:=+1$, the function $S_{\varepsilon}(x)$ then directly satisfies (2.4) and (2.6), and, as a short calculation shows, it also satisfies (2.5), up to an additive term $O(\varepsilon)$, i.e.,

$$
\frac{1}{3}+O\left(\varepsilon^{2}\right)=\left|S_{\varepsilon}\left(x_{j}\right)-i \operatorname{Im} \phi_{\varepsilon}\left(x_{j}\right)\right|=\left\|\operatorname{Im} \phi_{\varepsilon}\right\|_{I}+O(\varepsilon) \quad(j=1,2) .
$$

In addition, it can be similarly verified that

$$
\left\|S_{\varepsilon}-i \operatorname{Im} \phi_{\varepsilon}\right\|_{I}=\frac{1}{3}+O(\varepsilon) \text {, }
$$

and

$$
M:=\min _{j=1,2}\left\{\left|S_{\varepsilon}\left(x_{j}\right)+\operatorname{Re} \phi_{\varepsilon}\left(x_{j}\right)\right|\right\}=1+O\left(\varepsilon^{2}\right) .
$$

By (2.2) of Proposition 1, we thus have the upper bound

$$
\gamma_{0,2} \leq \frac{\left\|S_{\varepsilon}-i \operatorname{Im} \phi_{\varepsilon}\right\|_{I}}{M}=\frac{1}{3}+O(\varepsilon),
$$

for all $\varepsilon>0$ sufficiently small, whence on letting $\varepsilon \rightarrow 0$,

$$
\gamma_{0,2} \leq \frac{1}{3} \text {. }
$$

To establish the known upper bounds for $\gamma_{m, n}$ associated with the regions $R_{i}, i=1,2$, and 3, of Table 1, the authors of [1], [3], and [5] each, in essence, applied a variant of Proposition 1, with appropriate choices for $\phi_{\varepsilon}$ and $S_{\varepsilon}$, to obtain upper bounds for $\gamma_{m, n}$. Normalized forms of their choices of $\phi_{\varepsilon}$ and $S_{\varepsilon}$ are detailed in the next three theorems. For notation, $\prod_{j=1}^{m} d_{j}:=1$ when $m \leq 0$.

Theorem 1 (Trefethen and Gutknecht [5]). For any $m \geq 0$ and $\varepsilon>0$ sufficiently small, set

$$
g_{m, \varepsilon}(x):=\frac{\varepsilon \prod_{j=1}^{m}[-1+(2 j-1) \varepsilon-x]}{[x+(1+\varepsilon)]^{m+1}(i \sqrt{\varepsilon}-x)(1+\varepsilon-x)},
$$

so that $g_{m, \varepsilon} \in \pi_{m, m+3}^{c} \backslash \pi_{m, m+3}^{r}$, and set

$$
\phi_{m, \varepsilon}(x):=g_{m, \varepsilon}(x) /\left\|\operatorname{Im} g_{m, \varepsilon}(x)\right\|_{I}, \quad \text { and } \quad S_{\varepsilon}(x):=0 \text {. }
$$

Then, there is a constant $c>0$, independent of $\varepsilon$, such that for all $\varepsilon>0$ sufficiently small, there are $m+2$ distinct points $\left\{x_{j}(\varepsilon)\right\}_{j=1}^{m+2}$, with $-1 \leq x_{1}(\varepsilon)<$ $x_{2}(\varepsilon)<\cdots<x_{m+2}(\varepsilon) \leq 1$, for which

$$
\begin{gathered}
(-1)^{j} \operatorname{Re} \phi_{m, \varepsilon}\left(x_{j}(\varepsilon)\right) \geq c / \sqrt{\varepsilon} \quad(j=1,2, \ldots, m+2), \\
\left\|S_{\varepsilon}-i \operatorname{Im} \phi_{m, \varepsilon}\right\|_{I}=\left\|\operatorname{Im} \phi_{m, \varepsilon}\right\|_{I}=1,
\end{gathered}
$$

and

$$
M:=\min _{1 \leq j \leq m+2}\left|S_{\varepsilon}\left(x_{j}(\varepsilon)\right)+\operatorname{Re} \phi_{m, \varepsilon}\left(x_{j}(\varepsilon)\right)\right| \geq c / \sqrt{\varepsilon}
$$


Theorem 2 (Levin [1]). For any nonnegative integers $n$ and $k$ with $n \geq 2$ and $k$ even, set

$$
g_{k, n, \varepsilon}(x):=T_{k}(x) \cdot\left(\frac{x-i \varepsilon}{x+i \varepsilon}\right)^{n}
$$

where $T_{k}(x)$ is the normalized (i.e., $\left\|T_{k}\right\|_{I}=1$ ) Chebyshev polynomial of the first kind of degree $k$, and set $\phi_{k, n, \varepsilon}(x):=g_{k, n, \varepsilon}(x) /\left\|\operatorname{Im} g_{k, n, \varepsilon}(x)\right\|_{I}$ and $S_{\varepsilon}(x):=S_{k, n, \varepsilon}(x):=\operatorname{Re} \phi_{k, n, \varepsilon}(x)$. Then, there is a constant $c>0$, independent of $\varepsilon$, such that for all $\varepsilon>0$ sufficiently small, there are $k+2 n+1$ distinct points, $\left\{x_{j}(\varepsilon)\right\}_{j=1}^{k+2 n+1}$, with $-1 \leq x_{1}(\varepsilon)<x_{2}(\varepsilon)<\cdots<x_{k+2 n+1}(\varepsilon) \leq 1$, for which

$$
1-c \varepsilon \leq(-1)^{j+1} \operatorname{Re} \phi_{k, n, \varepsilon}\left(x_{j}(\varepsilon)\right) \leq\left\|S_{k, n, \varepsilon}-i \operatorname{Im} \phi_{k, n, \varepsilon}\right\|_{I} \leq 1+c \varepsilon,
$$

and

$$
M:=\min _{1 \leq j \leq k+2 n+1}\left|S_{k, n, \varepsilon}\left(x_{j}(\varepsilon)\right)+\operatorname{Re} \phi_{k, n, \varepsilon}\left(x_{j}(\varepsilon)\right)\right| \geq 2-2 c \varepsilon .
$$

Theorem 3 (Ruttan and Varga [3]). For any $m \geq 0$, let

$$
g_{m, \varepsilon}(x):=\frac{-2 \varepsilon i}{3} \sum_{j=0}^{m+1} \frac{\mu_{j}(-1)^{j}}{x-1+\frac{2 j}{m+1}-\varepsilon \mu_{j} i}
$$

where $\left\{\mu_{j}\right\}_{j=0}^{m+1}$ are any $m+2$ fixed positive numbers satisfying

$$
0<\mu_{j} \leq 1, \quad \sum_{j=0}^{m+1}(-1)^{j} \mu_{j}=0 \text { and } \sum_{j=0}^{m+1} j(-1)^{j} \mu_{j} \neq 0,
$$

so that $g_{m, \varepsilon} \in \pi_{m, m+2}^{c} \backslash \pi_{m, n+2}^{r}$, and let

$$
h(x)= \begin{cases}\frac{1-x^{2}}{1+x^{2}}, & x \in[-1,1] \\ 0, & \text { otherwise. }\end{cases}
$$

Set $\phi_{m, \varepsilon}(x):=g_{m, \varepsilon}(x) /\left\|\operatorname{Im} g_{m, \varepsilon}(x)\right\|_{I}$ and

$$
S_{\varepsilon}(x):=S_{m, \varepsilon}(x):=\left(\sum_{j=0}^{m+1}(-1)^{j} h\left(\frac{x-1+\frac{2 j}{m+1}}{\varepsilon}\right)\right) /\left\|\operatorname{Im} g_{m, \varepsilon}(x)\right\|_{I} .
$$

Then, there is a constant $c>0$, independent of $\varepsilon$ such that for all $\varepsilon>0$ sufficiently small, there are $m+2$ distinct points $\left\{x_{j}(\varepsilon)\right\}_{j=1}^{m+2}$, with $-1 \leq x_{1}(\varepsilon)<$ $x_{2}(\varepsilon)<\cdots<x_{m+2}(\varepsilon) \leq 1$, for which

$$
(-1)^{j} \delta \operatorname{Re} \phi_{m, \varepsilon}\left(x_{j}(\varepsilon)\right) \geq 2-c \varepsilon \quad(j=1,2, \ldots, m+2),
$$

where $\delta$ is a constant which is either +1 or -1 ,

$$
\left\|S_{m, \varepsilon}-i \operatorname{Im} \phi_{m, \varepsilon}\right\|<1+c \varepsilon,
$$


and

$$
M:=\min _{1 \leq j \leq m+2}\left|S_{m, \varepsilon}\left(x_{j}(\varepsilon)\right)+\operatorname{Re} \phi_{m, \varepsilon}\left(x_{j}(\varepsilon)\right)\right|>3-c \varepsilon
$$

On combining the results of (2.9) and (2.10) of Theorem 1 with (2.2) of Proposition 1, it is evident that $0 \leq \gamma_{m, m+3} \leq \sqrt{\varepsilon} / c$ for all $\varepsilon>0$ sufficiently small, so that (cf. Trefethen and Gutknecht [5])

$$
\gamma_{m, m+3}=0 \quad(m \geq 0)
$$

But as $\pi_{m, m+k}^{c} \supseteq \pi_{m, m+3}^{c}$ for all $k \geq 3$, the same function $\phi_{m, \varepsilon}$ of Theorem 1 can be used to deduce (as was pointed out in [5]) that

$$
\gamma_{m, n}=0 \quad(\text { all } n \geq m+3 ; m \geq 0) \text {. }
$$

In a similar fashion, on combining the results of Theorems 2 and 3 with Proposition 1 gives the upper bounds of

$$
\gamma_{m, n} \leq \frac{1}{2} \quad(m+1 \geq n \geq 1) ; \quad \gamma_{m, m+2} \leq \frac{1}{3} \quad(m \geq 0) .
$$

(We remark that the case $n=1$ of the first inequality of $(2.18)$ requires special handling. For details, see Levin [1].)

\section{OSCILlation OF THE REAL PART OF A RATIONAL FUNCTION}

For a given real or complex polynomial $p$, let $\partial p$ denote the exact degree of $p$. If $R=p / q$ is continuous on $[-1,1]$ where $p$ and $q$ are real polynomials, it is evident that $\operatorname{Re} R=R$ can have at most $\partial p$ sign changes (as, for example in (2.1)) since each sign change of $R$ corresponds to a zero of $p$. But, what can be said about the number of sign changes when $R=p / q$ is a continuous complex-valued rational function on $[-1,1]$ ? As we shall show in our next theorem, the number of possible sign changes of $\operatorname{Re} R$ depends not only on the degrees of $p$ and $q$, but also on the size of the oscillations of $\operatorname{Re} R$. For additional notation, let $\lfloor x\rfloor$ denote the greatest integer $N$ satisfying $N \leq x$. Then, we have the new result of

Theorem 4. Let $\phi=p / q$ be a complex rational function with no poles in $[-1,1]$ which satisfies $\|\operatorname{Im} \phi\|_{I} \leq 1$. Assume that there are real numbers $d>0$ and $\left\{x_{j}\right\}_{j=1}^{L}$, with $-1 \leq x_{1}<x_{2}<\cdots<x_{L} \leq 1$, for which

$$
\delta(-1)^{j} \operatorname{Re} \phi\left(x_{j}\right) \geq d \quad(j=1,2,3, \ldots, L),
$$

where $\delta$ is a constant which is either $=+1$ or -1 . If $\partial q \leq \partial p$ and if $d \geq 1$, then

$$
L \leq \partial p+1
$$

Similarly, if $\partial q>\partial p$, then

$$
L \leq \partial q \quad \text { whenever } d \geq 1,
$$


and

$$
L \leq\left\lfloor\frac{\partial p+\partial q+1}{2}\right\rfloor \text { whenever } d \geq 2 .
$$

The upper bounds for $L$ given in (3.2)-(3.4) are sharp in the following senses:

$$
\left\{\begin{array}{l}
\text { there exist rational functions, satisfying the ap- } \\
\text { propriate hypotheses, for which the upper bonds } \\
\text { for L given in (3.2)-(3.4) are attained (i.e., equal- } \\
\text { ity can hold in (3.2)-(3.4)) }
\end{array}\right.
$$

$$
\left\{\begin{array}{l}
\text { for any } d<1(d<2 \text { respectively }), \text { there exists } \\
\text { rational functions satisfying all but the hypothe- } \\
\text { ses on } d \text { in },(3.3)((3.4) \text { respectively) for which the } \\
\text { bound on } L \text { is exceeded. }
\end{array}\right.
$$

Proof. As the proofs associated with (3.5) and (3.6) are more direct, we first consider the sharpness results expressed in (3.5) and (3.6). To establish (3.5), we must exhibit rational function $\phi_{1}, \phi_{2}$, and $\phi_{3}$ which satisfy the hypotheses for (3.2), (3.3) and (3.4), respectively. To that end, first set $\phi_{1}=p / q$ where $q(x):=1$, and set $p(x):=T_{m}(x)=\cos (m \arccos x)($ for $-1 \leq x \leq 1)$. Then, $\partial p=m, 0=\left\|\operatorname{Im} \phi_{1}\right\|_{I} \leq 1$, and, with the known $m+1$ extremal points $\left\{\hat{x}_{j}:=\cos (j \pi / m)\right\}_{j=0}^{m}$ for the Chebyshev polynomial $T_{m}(x)$, (i.e., $T_{m}\left(\hat{x}_{j}\right)=$ $\left.(-1)^{j}\right)$, then $(3.1)$ is valid for $d=1$ and for the $L:=m+1$ points $\left\{\hat{x}_{j}\right\}_{j=0}^{m}$. In this case, equality then holds in (3.2). To verify that equality is attainable in (3.3), let $\phi_{2}=\phi_{m, \varepsilon}=p / q$ where $\phi_{m, \varepsilon}$ is given in Theorem 3. By Theorem 3, $\phi_{2}$ (an element of $\pi_{m, m+2}^{c} \backslash \pi_{m, m+2}^{r}$ ) satisfies (3.1) with $d \geq 1$ whenever $\varepsilon$ is sufficiently small. But $\partial p=m, \partial q=m+2$, and $L=m+2$, so, consequently, equality also can hold in (3.3).

It remains to verify that equality can hold in (3.4). Let $\phi=p / q$ be the rational function $\phi_{m, \varepsilon}$ given in Theorem 1. By (2.8), $\phi_{3}$ (an element of $\left.\pi_{m, m+3}^{c} \backslash \pi_{m, m+3}^{r}\right)$ satisfies (3.1) with $L=m+2$ and $d \geq 2$, provided $\varepsilon>0$ is sufficiently small. Since $\partial p=m$ and $\partial q=m+3$, we have $L=m+2=$ $\left\lfloor\frac{\partial p+\partial q+1}{2}\right\rfloor$, which shows that equality can hold in (3.4). This completes the proof of the sharpness in (3.5)

To establish the claimed sharpness (cf. (3.6)) of (3.4), consider first the function $\phi_{m, \varepsilon}$ (in $\pi_{m, m+2}^{c} \backslash \pi_{m, m+2}^{r}$ ) of Theorem 3. From Theorem 3, we see that $\phi_{m, \varepsilon}=p / q$ satisfies $\left\|\operatorname{Im} \phi_{m, \varepsilon}\right\|_{I} \leq 1$ and hypothesis (3.1) of Theorem 4 with $L=m+2$ and $d<2$ (for all $\varepsilon>0$ sufficiently small). But in this case, as $\partial p=m$, and as $\partial q=m+2$, then $L=m+2>\left\lfloor\frac{\partial p+\partial q+1}{2}\right\rfloor$, which shows that the inequality of (3.4) of Theorem 4 can fail if the condition $d \geq 2$ is deleted. In a similar constructive manner, using $\phi_{\varepsilon}(x)=\phi_{k, n, \varepsilon}(x) /(1+\varepsilon x)^{k+1}$ where $\phi_{k, n, \varepsilon}$ is defined in Theorem 2, one obtains the sharpness, as claimed in (3.6), for the inequality of (3.3). 


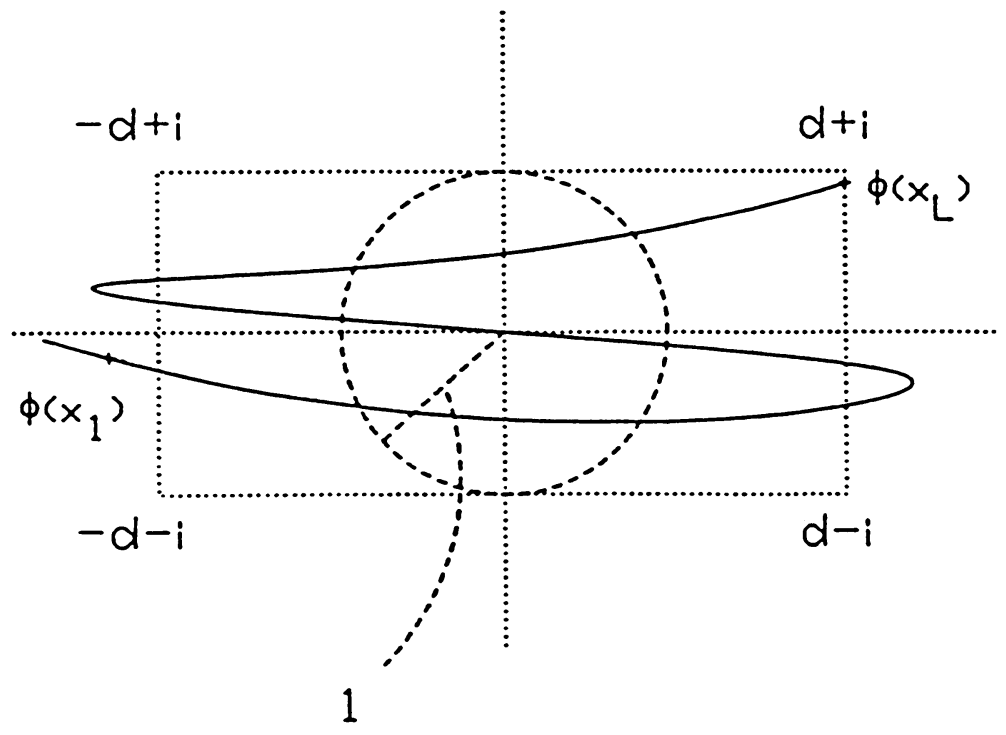

FIGURE 1

We now establish (3.2)-(3.4). We remark that inequalities (3.2) and (3.3) can be deduced from the results found in [1], but for completeness we include a proof here.

To establish (3.2) of Theorem 4, we use a geometrical argument, suggested by the work of Levin [1]. Assume $d \geq 1$, and consider a circle $C:=\{z:|z|=1\}$ and a rectangle $B$ with vertices $\pm d \pm i$ as indicated in Figure 1. Condition (3.1) and the assumption that $\|\operatorname{Im} \phi\|_{I} \leq 1$ imply that the curve (in the extended plane) $\Gamma_{1}:=\{z=\phi(x): x \in(-\infty, \infty)\}$ intersects the vertical sides of $B$, and, hence the circle $C$ in $2(L-1)$ points as $x$ increases from $x_{1}$ to $x_{L}$. (Here, points where $\Gamma_{1}$ is tangent to $C$ are counted twice.) If $x$ gives such an intersection of the curve $\Gamma_{1}$ and $C$, i.e.,

$$
|\phi(x)|^{2}=\left|\frac{p(x)}{q(x)}\right|^{2}=1,
$$

then $x$ is also a zero of the polynomial

$$
P(x):=|p(x)|^{2}-|q(x)|^{2} .
$$

The above discussion shows that there are at least $2(L-1)$ zeros of $P(x)$ in $\left[x_{1}, x_{L}\right]$.

If $\partial p \geq \partial q$, then $P(x)$ of (3.7) is a polynomial in $x$ with degree at most $2 \partial p$. Therefore, it must follow that $2(L-1) \leq \partial P(x) \leq 2 \partial p$, from which we obtain (3.2).

Next, to establish (3.3) of Theorem 4, assume that hypothesis (3.1) is valid, that $\partial q>\partial p$, and that $d \geq 1$. As in the previous case, we know that that 


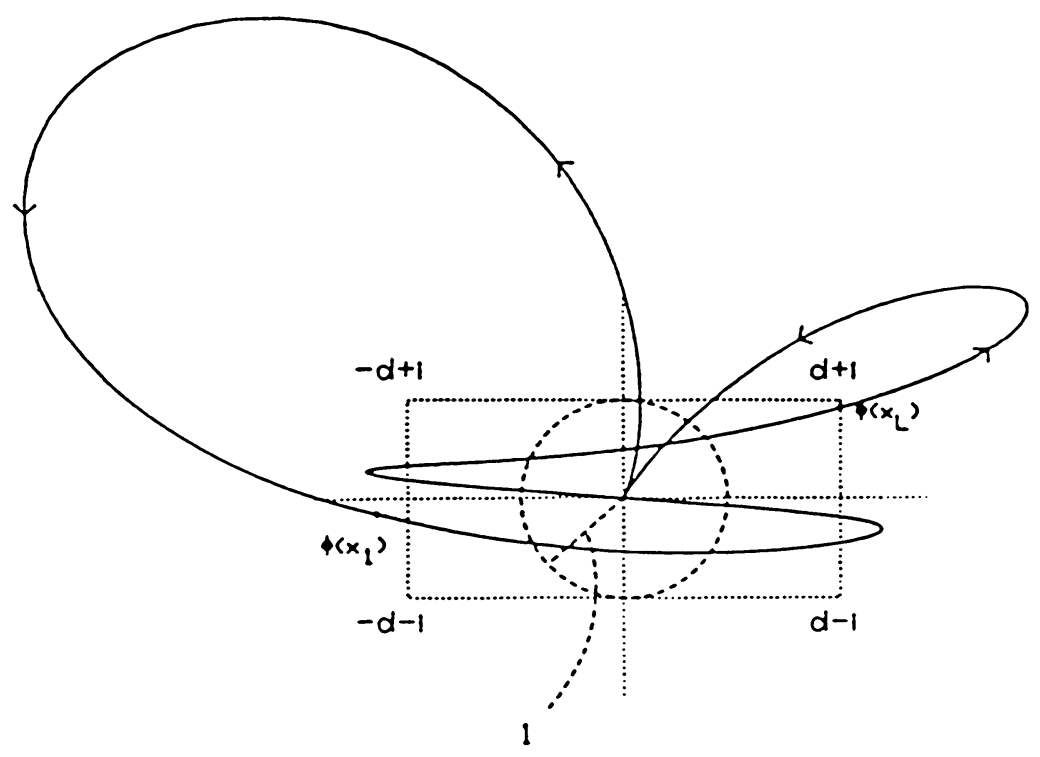

FigURE 2

portion of the curve $\Gamma_{1}$, as $x$ increases from $x_{1}$ to $x_{L}$, intersects the circle $C$ at least $2(L-1)$ times. Since $d \geq 1$, it is geometrically clear that $\phi\left(x_{1}\right)$ and $\phi\left(x_{L}\right)$ both lie outside of $C$ (cf. (3.1)) if any of the following statements is valid:

$$
\left\{\begin{aligned}
& \text { (i) } d>1 ; \\
& \text { (ii) } \delta(-1) \operatorname{Re} \phi\left(x_{1}\right)>1 \text { and } \delta(-1)^{L} \operatorname{Re} \phi\left(x_{L}\right)>1 ; \\
& \text { (iii) } \operatorname{Im} \phi\left(x_{1}\right) \neq 0 \neq \operatorname{Im} \phi\left(x_{L}\right) .
\end{aligned}\right.
$$

But, in this case (i.e., $\partial q>\partial p$ ), it follows that $\phi(x) \rightarrow 0$ as $x \rightarrow \pm \infty$. As 0 is an interior point of $C$, then there is evidently an additional intersection of $\Gamma_{1}$ and $C$ in each of the intervals $\left(-\infty, x_{1}\right)$ and $\left(x_{L},+\infty\right)$. (This is illustrated in Figure 2.) Thus, $P(x)$ of (3.7) must have a total of at least $2 L$ zeros. As $\partial q>\partial p$, then $\partial P=2 \partial q$, so that $2 L \leq 2 \partial q$. This establishes (3.3) whenever $\phi\left(x_{1}\right)$ and $\phi\left(x_{2}\right)$ both lie outside of $C$.

For the remaining case, suppose (in contrast with equations (3.8)) that $\delta(-1) \phi\left(x_{1}\right)=1=d$ and, for convenience, assume $\delta=+1$, so that $\phi\left(x_{1}\right)=$ -1 . If $\Gamma_{1}$ is not tangent to $C$ at -1 (this possibility is shown on the left of Figure 3), then it is possible to find a real $\tilde{x}_{1}$ sufficiently near $x_{1}$ for which $-\operatorname{Re} \phi\left(\tilde{x}_{1}\right)>1$ and $\|\operatorname{Im} \phi\|_{\left[\tilde{x}_{1},+1\right]} \leq 1$ are both satisfied. With a possible linear change in scale (mapping $\left[\tilde{x}_{1},+1\right]$ into $[-1,+1]$ ), then $\phi\left(\tilde{x}_{1}\right)$ is outside $C$, and the previous argument can be applied. Finally, if $\Gamma_{1}$ is tangent to $C$ at $x=1$ (as indicated on the right of Figure 3), this contact implies that $x=1$ is a zero of multiplicity at least two of $P(x)$, and we conclude in all cases that $P(x)$ must have at least $2 L$ zeros, which gives (3.3).

Now, for the remaining inequality (3.4) of Theorem 4, assume $\partial q>\partial p$ and $d \geq 2$. Again, consider the rectangle $B$ with vertices $\pm d \pm i$. The assumption 


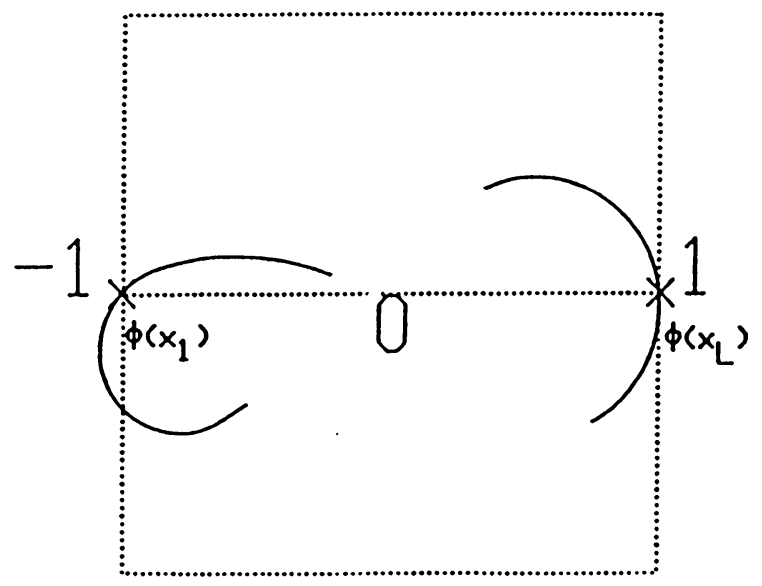

FIGURE 3

that $d \geq 2$ means that the circles $C_{1}:=\{z:|z+1|=1\}$ and $C_{2}:=\{z:|z-1|=$ $1\}$ each lie within the rectangle $B$. As in the cases above, condition (3.1) and the assumption that $\|\operatorname{Im} \phi\|_{I} \leq 1$, imply that that portion of the curve $\Gamma_{1}$ intersects $C_{1}$ in $2(L-1)$ points as $x$ increases from $x_{1}$ to $x_{L}$. (Again, points of tangency are counted twice). Let $\left\{v_{j}\right\}_{j=1}^{2(L-1)}$ be the $2(L-1)$ points with $x_{1} \leq v_{1} \leq v_{2} \leq \cdots \leq v_{2(L-1)} \leq x_{L}$ for which $\left\{\phi\left(v_{j}\right)\right\}_{j=1}^{2(L-1)}$ lie on $C_{1}$. Thus, the points $v_{1}, v_{2}, \ldots, v_{2(L-1)}$ satisfy

$$
\left|\phi\left(v_{j}\right)+1\right|^{2}=1 \quad(j=1,2, \ldots, 2(L-1)) .
$$

Similarly, we see that $\Gamma_{1}$ intersects $C_{2}$ in $2(L-1)$ points. Let $\left\{u_{j}\right\}_{j=1}^{2(L-1)}$ be the $2(L-1)$ points with $x_{1} \leq u_{1} \leq u_{2} \leq \cdots \leq u_{2(L-1)} \leq x_{L}$ for which $\left\{\phi\left(u_{j}\right)\right\}_{j=1}^{2(L-1)}$ lies on $C_{2}$. This situation is illustrated in Figure 4.

Currently, the polynomials $p$ and $q$ are determined only up to a multiplicative constant. So, without loss of generality, we may assume that

$$
p(x)=\prod_{j=1}^{\partial p}\left(x-\alpha_{j}\right) \quad \text { and } \quad q(x)=\beta \prod_{j=1}^{\partial q}\left(x-\beta_{j}\right) \quad(\beta \neq 0),
$$

where $\left\{\alpha_{j}\right\}_{j=1}^{\partial p}$ are the zeros of $\phi$ and $\left\{\beta_{j}\right\}_{j=1}^{\partial q}$ are the poles of $\phi$. With this representation, (3.9) implies that

$$
P_{1}(x):=|p(x)+q(x)|^{2}-|q(x)|^{2}=2 \operatorname{Re}\{\overline{p(x)} q(x)\}+|p(x)|^{2}
$$

has $2(L-1)$ zeros $\left\{v_{j}\right\}_{j=1}^{2(L-1)}$ in $\left[x_{1}, x_{L}\right]$. And similarly,

$$
P_{2}(x):=|p(x)-q(x)|^{2}-|q(x)|^{2}=-2 \operatorname{Re}\{\overline{p(x)} q(x)\}+|p(x)|^{2}
$$

has $2(L-1)$ zeros $\left\{u_{j}\right\}_{j=1}^{2(L-1)}$ in $\left[x_{1}, x_{L}\right]$. How the proof now proceeds depends on the sign of $\operatorname{Re} \beta$. If $\operatorname{Re} \beta<0$ we will find that $P_{1}(x)$ has enough 


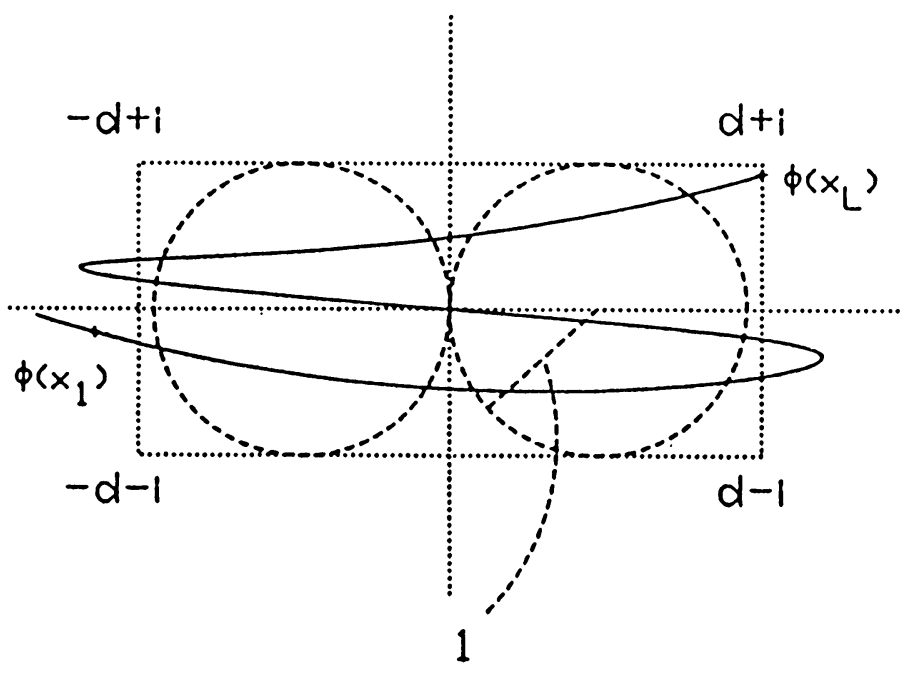

FIGURE 4

additional zeros to establish (3.4). If $\operatorname{Re} \beta \geq 0, P_{2}(x)$ can be used to establish (3.4). We treat only the case $\operatorname{Re} \beta \geq 0$, the case $\operatorname{Re} \beta<0$ being completely similar. Our goal is to find two additional zeros for $P_{2}(x)$ when $K:=\partial q-\partial p$ is an even positive integer and one additional zero when $K$ is an odd positive integer. Note, first, that if $\Gamma_{1}$ is tangent to $C_{2}$ at $x_{1}$, then $x_{1}$ is a zero of $P_{2}(x)$ with multiplicity at least 2 . In that case, we have an additional zero associated with $x_{1}$. In a similar fashion, we find an additional zero associate with $x_{L}$ if $\Gamma_{1}$ is tangent to $C_{2}$ at $x_{L}$.

There are three cases to consider: $K$ even and $\operatorname{Re} \beta>0, K$ odd and $\operatorname{Re} \beta>0$, and $\operatorname{Re} \beta=0$.

Case 1: $K$ even and $\operatorname{Re} \beta>0$. As we observed above, if $\Gamma_{1}$ is tangent to $C_{2}$ at $x_{1}$, then there is an additional zero of $P_{2}(x)$ associated with $x_{1}$. If $\Gamma_{1}$ is not tangent to $C_{2}$ at $x_{1}$, then since $\left|\operatorname{Re} \phi\left(x_{1}\right)\right| \geq d \geq 2$ we proceed as in the proof of (3.3) to show that there is a real $\tilde{x}_{1}$ arbitrarily near $x_{1}$ (and possibly equal to $\left.x_{1}\right)$ for which $\operatorname{sgn} \operatorname{Re} \phi\left(x_{1}\right)=\operatorname{sgn} \operatorname{Re} \phi\left(\tilde{x}_{1}\right)$ and $\left|\operatorname{Re} \phi\left(\tilde{x}_{1}\right)\right|>d$. If one replaces $x_{1}$ with $\tilde{x}_{1}$, then the hypotheses of the theorem still hold (after a possible linear change in scale). Therefore, without loss of generality, we may assume $\left|\operatorname{Re} \phi\left(x_{1}\right)\right|>d \geq 2$, and hence $\left|\phi\left(x_{1}\right)\right|=\left|\frac{p\left(x_{1}\right)}{q\left(x_{1}\right)}\right|>d \geq 2$. Consequently, it follows that

$$
\left|\operatorname{Re} \frac{q\left(x_{1}\right)}{p\left(x_{1}\right)}\right| \leq\left|\frac{q\left(x_{1}\right)}{p\left(x_{1}\right)}\right|<\frac{1}{2}
$$

Using the (3.10), we see that

$$
\operatorname{Re} \frac{q(x)}{p(x)}=(\operatorname{Re} \beta) x^{K}+\text { lower order terms in } x .
$$


Since $\operatorname{Re} \beta>0$ and $K$ is an even positive integer, then as $x \rightarrow-\infty,(3.14)$ shows that $\operatorname{Re} \frac{q(x)}{p(x)} \rightarrow+\infty$. This together with (3.13) establishes that there is an $\hat{x}$ in $\left(-\infty, x_{1}\right)$ for which

$$
\operatorname{Re} \frac{q(\hat{x})}{p(\hat{x})}=\frac{1}{2}
$$

But (3.15) may be rewritten as

$$
-2 \operatorname{Re}\{\overline{p(\hat{x})} q(\hat{x})\}+|p(\hat{x})|^{2}=0,
$$

which shows that $P_{2}(x)$ has a zero in $\left(-\infty, x_{1}\right)$, when $\Gamma_{1}$ is not tangent to $C_{2}$. So, in either case ( $\Gamma_{1}$ tangent to $C_{2}$, or $\Gamma_{1}$ not tangent to $\left.C_{2}\right)$, we find an extra zero associated with $x_{1}$. Similarly, we find an extra zero associated with $x_{L}$. Thus, when $K$ is an even positive integer and $\operatorname{Re} \beta>0, P_{2}(x)$ has $2 L$ zeros. But then,

$$
2 L \leq \partial P_{2} \leq \partial p+\partial q \leq \partial p+\partial q+1
$$

Hence,

$$
L \leq\left\lfloor\frac{\partial p+\partial q+1}{2}\right\rfloor \text {, }
$$

which establishes (3.4) for this case.

Case 2: $K$ odd and $\operatorname{Re} \beta>0$. If $\Gamma_{1}$ is tangent to $C_{2}$ at $x_{L}$, the tangency then gives the required additional zero. When $\Gamma_{1}$ is not tangent to $C_{2}$ at $x_{L}$ then, after a possible substitution of $x_{L}$ with a point $\tilde{x}_{L}$ sufficiently close to $x_{L}$, followed by a possible linear substitution, we find that $\left|\phi\left(x_{L}\right)\right|>2$, from which

$$
\left|\operatorname{Re} \frac{q\left(x_{L}\right)}{p\left(x_{L}\right)}\right|<\frac{1}{2}
$$

follows. As $\operatorname{Re} \beta>0$, (3.14) shows that $\operatorname{Re} \frac{q(x)}{p(x)} \rightarrow+\infty$ as $x \rightarrow+\infty$. Arguing as in Case 1, this together with (3.16) yields that $P_{2}(x)$ has an additional zero in $\left(x_{L},+\infty\right)$. Thus, we find that $P_{2}(x)$ has $2 L-1$ zeros, and therefore

$$
2 L \leq \partial P_{2}+1 \leq \partial p+\partial q+1 \text {. }
$$

Consequently,

$$
L \leq\left\lfloor\frac{\partial p+\partial p+1}{2}\right\rfloor
$$

Case 3: $\operatorname{Re} \beta=0$. Since $\partial q>\partial p$, it follows from (3.10) and (3.12) that

$$
\begin{aligned}
P_{2}(x) & =-2 \operatorname{Re}(\overline{p(x)} q(x))+|p(x)|^{2} \\
& =-2(\operatorname{Re} \beta) x^{\partial q+\partial p}+(\operatorname{Re} \mu) x^{\partial q+\partial p-1}+\text { lower order terms, }
\end{aligned}
$$

for some constant $\mu$. But, as $\operatorname{Re} \beta=0$, we have that $2 L-2 \leq \partial P_{2} \leq$ $\partial p+\partial q-1$. Therefore

which gives (3.4).

$$
L \leq\left\lfloor\frac{\partial p+\partial q+1}{2}\right\rfloor \text {, }
$$




\section{LOWER BOUNDS FOR $\gamma_{m, n}$}

With the aid of Theorem 4, we will now establish that $\gamma_{m, m+2}=1 / 3$ for all $m \geq 0$, and show that the previously mentioned lower bounds for $\gamma_{m, n}, m \geq$ $n+2$, hold.

Theorem 5. Let $(m, n)$ be a pair on nonnegative integers with $n \geq 1$, let $f \in C^{r}[-1,1] \backslash \pi_{m, n}^{r}$, and let $r$ and $R$ be respectively the best uniform approximation of $f$ on $[-1,1]$ from $\pi_{m, n}^{r}$ and $\pi_{m, n}^{c}$. Then,

$$
\|f-R\|_{I} /\|f-r\|_{I}>1 / 2 \quad \text { if } m+1 \geq n
$$

and

$$
\|f-R\|_{I} /\|f-r\|_{I}>1 / 3 \quad \text { if } m+2 \geq n \text {. }
$$

Hence,

$$
\gamma_{m, n}=1 / 2 \quad \text { if } m+1 \geq n,
$$

and

$$
\gamma_{m, n}=1 / 3 \quad \text { if } m+2=n .
$$

Proof. Let $S:=\|f-R\|_{I} /\|f-r\|_{I}$. Set $e:=f-r, R:=p_{1} / q_{1}$, and $r:=p_{2} / q_{2}$ where the pairs $\left(p_{1}, q_{1}\right)$ and $\left(p_{2}, q_{2}\right)$ are assumed to have no common factors. Since $f \notin \pi_{m, n}^{r}$, then by multiplying $f, r$, and $R$ by an appropriate constant, we may assume that $\|e\|_{I}=1$. As $r$ is the best uniform approximant of $f$, there exist at least $L:=m+n+2-\min \left(m-\partial p_{2} ; n-\partial q_{2}\right)$ distinct points $\left\{x_{j}\right\}_{j=1}^{L}$, with $-1 \leq x_{1}<x_{2}<\cdots<x_{L} \leq 1$, such that $e\left(x_{j}\right)=(-1)^{j} \delta$ for all $1 \leq j \leq L$, where $\delta$ is a constant which is either +1 or -1 . Again, on multiplying by -1 , if necessary, we may take $\delta=1$, i.e., $e\left(x_{1}\right)=-1$.

With this normalization,

$$
S:=\|f-R\|_{I} \geq\left|f\left(x_{j}\right)-R\left(x_{j}\right)\right|=\left|(-1)^{j}+r\left(x_{j}\right)-R\left(x_{j}\right)\right| \quad(1 \leq j \leq L),
$$

which is possible only if

$$
(-1)^{j} \operatorname{Re}\left(R\left(x_{j}\right)-r\left(x_{j}\right)\right) \geq 1-S \quad(1 \leq j \leq L) .
$$

Let $\phi(x):=(R(x)-r(x)) / S:=p(x) / q(x)$ where $p$ and $q$ are polynomials with no common factors. Then, as

$$
S=\|f-R\|_{I}=\|e-R+r\|_{I} \geq\|\operatorname{Im}(e-R+r)\|_{I}=\|\operatorname{Im} R\|_{I},
$$

(4.5) implies

$$
(-1)^{j} \operatorname{Re} \phi\left(x_{j}\right) \geq \frac{1-S}{S}=: d \quad(1 \leq j \leq L),
$$

and (4.6) implies

$$
\|\operatorname{Im} \phi\|_{I} \leq 1
$$


To establish (4.1) of Theorem 5, it suffices to establish the contrapositive of (4.1) i.e., if $S \leq \frac{1}{2}$, then $m+1<n$, or equivalently

$$
\text { if } S \leq \frac{1}{2} \text {, then } m+2 \leq n \text {. }
$$

Similarly, to establish (4.2) of Theorem 5, it suffices to establish that if $S \leq \frac{1}{3}$, then $m+2<n$, or equivalently

$$
\text { if } S \leq \frac{1}{3} \text {, then } m+3 \leq n \text {. }
$$

To this end, first assume that $S \leq 1 / 2$. Then from (4.7), $d \geq 1$, and on applying Theorem 4 to (4.7) and (4.8), we obtain

$$
L \leq \partial p+1 \text { if } \partial p \geq \partial q
$$

and

$$
L \leq \partial q \quad \text { if } \partial p<\partial q
$$

Since

$$
\begin{aligned}
\phi(x) & =p(x) / q(x)=(R(x)-r(x)) / S \\
& =\frac{p_{1}(x) q_{2}(x)-p_{2}(x) q_{1}(x)}{S q_{1}(x) q_{2}(x)}
\end{aligned}
$$

it follows that

$$
\left\{\begin{array}{l}
\partial p \leq \max \left(\partial p_{1}+\partial q_{2} ; \partial p_{2}+\partial q_{1}\right), \text { and } \\
\partial q=\partial q_{1}+\partial q_{2}
\end{array}\right.
$$

If $\partial p \geq \partial q$, then (4.11) holds and thus

$$
\begin{aligned}
m+n+2 & -\min \left(m-\partial p_{2} ; n-\partial q_{2}\right) \\
= & : L \leq \partial p+1 \leq \max \left(\partial p_{1}+\partial q_{2}, \partial p_{2}+\partial q_{1}\right)+1
\end{aligned}
$$

But, we claim that (4.14) is impossible for any $m, n, \partial q_{1}, \partial q_{2}, \partial p_{1}$, and $\partial p_{2}$ with $n \geq \partial q_{1} \geq 0, n \geq \partial q_{2} \geq 0, m \geq \partial p_{1}$, and $m \geq \partial p_{2}$. To see this, suppose that $\max \left(\partial p_{1}+\partial q_{2} ; \partial p_{2}+\partial q_{1}\right)=\partial p_{1}+\partial q_{2}$. In this case, (4.14) becomes

$$
m+n+2-\min \left(n-\partial q_{2} ; m-\partial p_{2}\right) \leq \partial p_{1}+\partial q_{2}+1 \text {, }
$$

or equivalently

$$
\left\{m-\partial p_{1}\right\}+\left\{\left(n-\partial q_{2}\right)-\min \left(n-\partial q_{2} ; m-\partial p_{2}\right)\right\} \leq-1,
$$

which is impossible as each term in braces on the left side of (4.15) is nonnegative. A similar argument gives a contraction if it is assumed that

$$
\max \left\{\partial p_{1}+\partial q_{2}+\partial q_{1}\right\}=\partial p_{2}+\partial q_{1}
$$

Therefore, it follows that $\partial q>\partial p$.

With $\partial q>\partial p,(4.12)$ implies from (4.13) that

$$
L:=m+n+2-\min \left(n-\partial q_{2} ; m-\partial p_{2}\right) \leq \partial q=\partial q_{1}+\partial q_{2},
$$

or

$$
\left\{\left(n-\partial q_{1}\right)\right\}+\left\{\left(n-\partial q_{2}\right)-\min \left(n-\partial q_{2} ; m-\partial p_{2}\right)\right\} \leq n-(m+2)
$$


Because each term in braces on the left side of (4.16) is nonnegative, we conclude that $0 \leq n-(m+2)$, which establishes (4.9).

Now, assume $S \leq 1 / 3$. Then $d \geq 2$ from (4.7), and (4.8) and Theorem 4 combine to give

$$
L \leq \partial p+1 \quad \text { if } \partial p \geq \partial q
$$

and

$$
L \leq\left\lfloor\frac{\partial p+\partial q+1}{2}\right\rfloor \quad \text { if } \partial q>\partial p .
$$

Arguing as above, it similarly follows that assuming $\partial p \geq \partial q$ leads to a contradiction. This leaves only the possibility that $\partial q>\partial p$. Using (4.18), we then have

$$
L:=m+n+2-\min \left(m-\partial p_{2} ; n-\partial q_{2}\right) \leq\left\lfloor\frac{\partial p+\partial q+1}{2}\right\rfloor .
$$

Inequality (4.19) then implies

$$
2 m+2 n+4-2 \min \left(m-\partial p_{2} ; n-\partial q_{2}\right) \leq \partial p+\partial q+1,
$$

and on using (4.13), we have that

$$
\begin{aligned}
2 m+ & 2 n+4-2 \min \left(n-\partial q_{2} ; m-\partial p_{2}\right) \\
& \leq \max \left(\partial p_{1}+\partial q_{2} ; \partial p_{2}+\partial q_{1}\right)+\partial q_{1}+\partial q_{2}+1 .
\end{aligned}
$$

If $\max \left(\partial p_{1}+\partial q_{2} ; \partial p_{2}+\partial q_{1}\right)=\partial p_{1}+\partial q_{2}$, then (4.20) may be rewritten as

$$
\begin{aligned}
& \left\{m-\partial p_{1}\right\}+2\left\{\left(n-\partial q_{2}\right)-\min \left(n-\partial q_{2} ; m-\partial p_{2}\right)\right\} \\
& \leq \partial q_{1}-(m+3) \leq n-(m+3) .
\end{aligned}
$$

But, as each term in braces on the left side of (4.21) is nonnegative, it is clear that (4.10) holds in this case. A similar argument establishes (4.10) when it is assumed that $\max \left(\partial p_{1}+\partial q_{2} ; \partial p_{2}+\partial q_{1}\right)=\partial p_{2}+\partial q_{1}$.

To complete the proof of Theorem 5, we see that (4.1) implies

$$
\gamma_{m, n} \geq 1 / 2 \quad \text { if } m+1 \geq n \geq 1,
$$

while the reverse inequality holds from (2.18). Thus, we have

$$
\gamma_{m, n}=1 / 2 \text { if } m+1 \geq n \geq 1,
$$

the desired result of (4.3). Similarly, (4.2) implies

$$
\gamma_{m, m+2} \geq 1 / 3 \text { for any } m \geq 0 \text {, }
$$

while the reverse inequality holds from (2.18). Thus, we have

$$
\gamma_{m, m+2}=1 / 3 \text { for any } m \geq 0,
$$

the desired result of (4.4).

Remark. We note that Trefethen and Gutknecht conjectured in [5] that $\gamma_{m, n}$ could only be zero if $m \leq n+3$. Theorem 5 thus establishes the validity of their conjecture! 


\section{REFERENCES}

1. A. Levin, On the degree of complex rational approximation to real functions, Constructive Approximation 2 (1986), 213-219.

2. G. Meinardus, Approximation of functions: Theory and numerical methods, Springer-Verlag New York.

3. A. Ruttan and R. S. Varga, Real vs. complex rational Chebyshev approximation on an interval: $\gamma_{m, m+2} \leq 1 / 3$, Rocky Mountain J. (to appear).

4. E. B. Saff and R. S. Varga, Nonuniqueness of best complex rational approximations to real functions on real intervals, J. Approximation Theory 23 (1978), 78-85.

5. L. N. Trefethen and M. H. Gutknecht, Real vs. complex rational Chebyshev approximation on an interval, Trans. Amer. Math. Soc. 280 (1983), 555-561.

6. R. S. Varga, Topics in polynomial and rational interpolation and approximation, Univ. of Montreal Press, Montreal.

Department of Mathematical Sciences, Kent State University, Kent, Ohio 44242 